\title{
WEAK CONVERGENCE AND WEAK COMPACTNESS IN ABSTRACT $M$ SPACES
}

\author{
SHUTAO CHEN AND HUIYING SUN \\ (Communicated by Palle E. T. Jorgensen)
}

\begin{abstract}
This paper presents some properties of bounded linear functionals on $\sigma$ complete abstract $M$ spaces, from which some criteria for weak convergence and weak compactness in such spaces are obtained.
\end{abstract}

\section{Abstract $M$ SPACES AND ABSTRACT $L$ SPACES}

Definition $1[1,2]$. Let $X$ be a Banach lattice.

(1) $X$ is called an abstract $M$ space $(X \in A M)$ if $x \wedge y=0$ implies

$$
\|x+y\|=\max \{\|x\|,\|y\|\} \text {. }
$$

(2) $X$ is called an abstract $L$ space $(X \in A L)$ if $x \wedge y=0$ implies

$$
\|x+y\|=\|x\|+\|y\| .
$$

For a Banach lattice $X$ and $x \in X, f, g \in X^{*}$, as in [1], we define

$$
\begin{array}{cc}
(f \vee g)(x)=\sup \{f(u)+g(x-u): 0 \leq u \leq x\} & (x \geq 0), \\
(f \wedge g)(x)=\inf \{f(u)+g(x-u): 0 \leq u \leq x\} & (x \geq 0) .
\end{array}
$$

Then by Theorem 118.1 and 118.5 in [3], we have

Lemma 2. Let $X$ be a Banach lattice. Then

(1) $X \in A M$ implies $X^{*} \in A L$ and $X \in A L$ implies $X^{*} \in A M$;

(2) $X \in A M$ iff for any $x, y \in X, x, y \geq 0$ implies

$$
\|x \vee y\|=\max \{\|x\|,\|y\|\} ;
$$

(3) $X \in A L$ iff for any $x, y \in X, x, y \geq 0$ implies

$$
\|x+y\|=\|x\|+\|y\| .
$$

Let $X$ be a lattice and $x, u, v \in X$ and $u \geq 0, v \geq 0$. By Theorem 11.8 and 11.10 in [2], if $x=u-v$, then $u=x^{+}+u \wedge v$ and $v=x^{-}+u \wedge v$, where $x^{+}=x \vee 0$ and $x^{-}=(-x) \vee 0$. Especially, if $u \wedge v=0$, then $u=x^{+}$ and $v=x^{-}$. If $X \in A L$, then $\|x\|=\left\|x^{+}\right\|+\left\|x^{-}\right\|$and by Lemma 2, $\|u\|=$ $\left\|x^{+}\right\|+\|u \wedge v\|,\|v\|=\left\|x^{-}\right\|+\|u \wedge v\|$. Hence, we have

Received by the editors June 21, 1993 and, in revised form, August 9, 1993.

1991 Mathematics Subject Classification. Primary 46B42.

Key words and phrases. Banach lattice, abstract $M$ spaces, weak convergence, weak compactness, extreme point.

The authors are supported by the National Science Foundation of China and the Heilongjiang Science Foundation. 
Lemma 3. Let $X \in A L$ and $x \in X$. Then the above decomposition $x=x^{+}-x^{-}$ is unique in the sense that if $x=u-v, u \geq 0, v \geq 0$, and $\|u\|+\|v\|=\|x\|$, then $u=x^{+}$and $v=x^{-}$.

For a subset $E$ of a Banach lattice $X$ and $x \in X$, we write

$$
E^{\perp}=\{x \in X: x \perp y \text { for all } y \in E\}, \quad x^{\perp}=\{x\}^{\perp},
$$

where $x \perp y$ means $|x| \wedge|y|=0$. If $x \in X=E+E^{\perp}$, then $x$ can be uniquely decomposed into $x=u+v$, where $u \in E$ and $v \in E^{\perp}$. In this case, we write $\left.x\right|_{E}=u$ and $\left.f\right|_{E}(x)=f(u)$ for $f \in X^{*}$.

Definition 4. Let $X$ be a Banach lattice. Then

(a) $X$ is said to be $\sigma$ complete, if for every order bounded sequence $\left\{x_{n}\right\}$ in $X, \bigvee_{n \geq 1}\left(x_{n}\right)$ exists in $X$.

(b) $X$ is said to be bounded $\sigma$ complete, provided that any norm bounded and order monotone sequence in $X$ is order convergent.

Clearly, bounded $\sigma$ complete Banach lattices are $\sigma$ complete. The inverse does not hold; for instance, $c_{0}$ is $\sigma$ complete but not bounded $\sigma$ complete. Moreover, according to [1], the space $C(K)$ of all continuous functions on a compact Hausdorff topological space $K$ is $\sigma$ complete if and only if $K$ is basically disconnected, i.e., the closure of every open $F_{\sigma}$ subset of $K$ is an open set.

For more detail about Banach lattices, also see [4] and [5].

\section{BOUNDED LINEAR FUNCTIONALS ON ABSTRACT $M$ SPACES}

For a Banach space $X$, we always denote by $B(X)$ and $S(X)$ the unit ball and the unit sphere of $X$ respectively.

Theorem 5. Let $X \in A M$ be $\sigma$ complete and $f \in X^{*}$. Then for any $\varepsilon>0$, there exists a subspace $E$ of $X$ such that $X=E+E^{\perp}$ and $\left\|\left.f^{+}\right|_{E^{\perp}}\right\|<\varepsilon$, $\left\|f^{-} \mid E\right\|<\varepsilon$.

Proof. Pick $x \in S(X)$ satisfying $f(x)>\|f\|-\varepsilon$, and set $E=\left(x^{-}\right)^{\perp}$. Then $x^{+} \in E, x^{-} \in E^{\perp}$, and by [1] $X=E+E^{\perp}$. Moreover, by Lemma 2,

$$
\begin{aligned}
& \left\|\left.f^{+}\right|_{E}\right\|+\left\|\left.f^{+}\right|_{E^{\perp}}\right\|+\left\|\left.f^{-}\right|_{E}\right\|+\left\|\left.f^{-}\right|_{E^{\perp}}\right\| \\
& \quad=\left\|f^{+}\right\|+\left\|f^{-}\right\|=\|f\|<f(x)+\varepsilon \\
& \quad=\left.f^{+}\right|_{E}(x)+\left.f^{+}\right|_{E^{\perp}}(x)-\left.f^{-}\right|_{E}(x)-\left.f^{-}\right|_{E^{\perp}}(x)+\varepsilon .
\end{aligned}
$$

Since $\left.f^{+}\right|_{E^{\perp}}(x) \leq 0$ and $\left.f^{-}\right|_{E}(x) \geq 0$, we find

$$
\begin{aligned}
& \left\|\left.f^{+}\right|_{E^{\perp}}\right\|+\left\|\left.f^{-}\right|_{E}\right\| \\
& \quad=\left\|f^{+}\right\|-\left\|\left.f^{+}\right|_{E}\right\|+\left\|f^{-}\right\|-\left\|\left.f^{-}\right|_{E^{\perp}}\right\| \\
& \quad \leq\left\|f^{+}\right\|-\left.f^{+}\right|_{E}(x)+\left\|f^{-}\right\|-\left.f^{-}\right|_{E^{\perp}}(x) \\
& \quad<\left.f^{+}\right|_{E^{\perp}}(x)-\left.f^{-}\right|_{E}(x)+\varepsilon \leq \varepsilon .
\end{aligned}
$$

Theorem 6. If a Banach lattice $X$ is bounded $\sigma$ complete and $B(X)$ is order closed, then every positive $f \in X^{*}$ (i.e., $f \geq 0$ ) is norm attainable, i.e., there exists $x \in S(X)$ satisfying $f(x)=\|f\|$.

Proof. Pick $x_{n}(\geq 0) \in S(X)$ such that $f\left(x_{n}\right) \rightarrow\|f\|$. Since $X$ is bounded $\sigma$ complete and $B(X)$ is order closed, $y=\bigvee_{n}\left(x_{n}\right)$ exists in $X$ and $\|y\|=1$. Hence, $y \geq x_{n} \geq 0$ and $f \geq 0$ implies $\|f\| \geq f(y) \geq f\left(x_{n}\right) \rightarrow\|f\|$. 
Remark. If $X \in A M$ is not bounded $\sigma$ complete, then the conclusion of Theorem 6 may be false. For instance, if $X=c_{0}$ and $f=\left(c_{n}\right) \in l_{1}$ with infinitely many $c_{n} \neq 0$, then $f$ is not norm attainable.

If $B(X)$ is not order closed, then the statement of the theorem is not necessarily true. For example, take $X=l_{\infty}$ and define

$$
|||x|||=\sup _{n \geq 1}\left\{\left|x_{n}\right|, k \cdot \limsup _{i \rightarrow \infty}\left|x_{i}\right|\right\}, \quad x=\left(x_{n}\right) \in l_{\infty},
$$

where $k>1$ is a constant. Then the norm $\|\cdot\| \|$ satisfies $\|x\|_{\infty} \leq\|\| x \| \mid \leq$ $k\|x\|_{\infty}$ for all $x \in l_{\infty}$ and $\|x\|_{\infty}=\|x\| \|$ for all $x \in c_{0}$. But for any bounded linear functional $f=\left(c_{n}\right) \in l_{1}$ on $l_{\infty}$ with infinitely many $c_{n} \neq 0, f$ cannot attain its norm on $B\left(l_{\infty},|| \cdot|| \mid\right)$.

Theorem 7. Let $X \in A M$ be bounded $\sigma$ complete and $B(X)$ order closed. Then $f \in X^{*}$ is norm attainable iff there exists a subspace $E$ of $X$ such that $f^{+}=\left.f\right|_{E}, f^{-}=-\left.f\right|_{E^{\perp}}$.

Proof. Sufficiency. By Theorem 6, there exist $x, y(\geq 0) \in S(X)$ such that $f^{+}(x)=\left\|f^{+}\right\|$and $f^{-}(y)=\left\|f^{-}\right\|$. Since $f^{+}=\left.f\right|_{E}$ and $f^{-}=-\left.f\right|_{E^{\perp}}$, we may assume $x \in E$ and $y \in E^{\perp}$ (otherwise we replace $x, y$ by $\left.x\right|_{E},\left.y\right|_{E^{\perp}}$ respectively). Let $u=x-y$. Then $\|u\|=\|x-y\|=\max \{\|x\|,\|y\|\}=1$ and hence, Lemma 2 implies

$$
\begin{aligned}
\|f\| & =\left\|f^{+}\right\|+\left\|f^{-}\right\|=f^{+}(x)+f^{-}(y) \\
& =\left.f\right|_{E}(x)+\left.f\right|_{E^{\perp}}(-y)=f(u) .
\end{aligned}
$$

Necessity. Choose $x \in S(X)$ satisfying $f(x)=\|f\|$, and define $E=\left(x^{-}\right)^{\perp}$. Then $X=E+E^{\perp}$ and $x^{+} \in E, x^{-} \in E^{\perp}$. Observe that $\|f\|=\left\|\left.f\right|_{E}\right\|+$ $\left\|\left.f\right|_{E^{\perp}}\right\|$; to prove $f^{+}=\left.f\right|_{E}$ and $f^{-}=-\left.f\right|_{E^{\perp}}$, it suffices to show $\left.f\right|_{E} \geq 0$ and $-\left.f\right|_{E^{\perp}} \geq 0$ thanks to Lemma 3. Indeed, if $\left.f\right|_{E}(y)<0$ for some $y(\geq 0)$ $\in S(X)$, then we may assume $y \in E$. Therefore, $z=-x^{-}-y$ satisfies $\|z\|=\max \left\{\left\|x^{-}\right\|,\|y\|\right\}=1$ and thus,

$$
\begin{aligned}
\left\|f^{-}\right\| & \geq f^{-}(-z)=f(z)-f^{+}(z) \geq f(z) \\
& =\left.f\right|_{E^{\perp}}\left(-x^{-}\right)-\left.f\right|_{E}(y)>\left.f\right|_{E^{\perp}}\left(-x^{-}\right)=-\left.f\right|_{E^{\perp}}(x) .
\end{aligned}
$$

Since $\left\|f^{+}\right\| \geq f\left(\left.x\right|_{E}\right)=\left.f\right|_{E}(x)$, this leads to a contradiction that

$$
\|f\|=\left\|f^{+}\right\|+\left\|f^{-}\right\|>\left.f\right|_{E}(x)-\left.f\right|_{E^{\perp}}(x)=f(x)=\|f\| .
$$

Similarly, we can verify $-\left.f\right|_{E^{\perp}} \geq 0$.

Definition 8 [6]. Let $X$ be a Banach space. $x \in S(X)$ is called an extreme point of $B(X)$ if $x=\lambda y+(1-\lambda) z, y, z \in B(X)$ and $\lambda \in(0,1)$, imply $y=z$. In this case, we write $x \in \operatorname{ext} B(X)$.

Since by the Rainwater Theorem [6], $x_{n} \rightarrow 0$ weakly in a Banach space $X$ iff $\left\{x_{n}\right\}$ is bounded, and $f\left(x_{n}\right) \rightarrow 0$ for every $f \in \operatorname{ext} B\left(X^{*}\right)$, we are encouraged to investigate the extreme points of the unit ball of a dual space.

Theorem 9. Let $X \in A M$ be $\sigma$ complete and $f \in S\left(X^{*}\right)$. Then $f \in \operatorname{ext} B\left(X^{*}\right)$ iff $f(x) f(y)=0$ for all $x, y \in X$ satisfying $x \wedge y=0$.

Proof. Sufficiency. First we show $\left\|f^{+}\right\|\left\|f^{-}\right\|=0$. In fact, for any $\varepsilon>0$, by Theorem 5 , there exist two orthogonal subspaces $E, F$ of $X$ such that 
$X=E+F$ and $\left\|\left.f^{-}\right|_{E}\right\|<\varepsilon,\left\|\left.f^{+}\right|_{F}\right\|<\varepsilon$. Choose $x \in S(X)$ such that $f(x)>\|f\|-\varepsilon$, and let $x=u+v$, where $u \in E$ and $v \in F$. Then $f(u) f(v)=0$ since $u \wedge v=0$. If $f(v)=0$, then

$$
\begin{aligned}
\|f\|-\varepsilon & <f(x)=\left.f^{+}\right|_{E}(u)-\left.f^{-}\right|_{E}(u) \\
& \leq\left\|\left.f^{+}\right|_{E}\right\|+\left\|\left.f^{-}\right|_{E}\right\|<\left\|f^{+}\right\|+\varepsilon .
\end{aligned}
$$

Letting $\varepsilon \rightarrow 0$, we find $\left\|f^{-}\right\|=\|f\|-\left\|f^{+}\right\|=0$. Similarly, if $f(u)=0$. Then $\left\|f^{+}\right\|=0$. Hence, without loss of generality, we may assume $f=f^{+}$.

Let $g, h \in S\left(X^{*}\right)$ satisfy $2 f=g+h$. Then $2 f=\left(g^{+}+h^{+}\right)-\left(g^{-}+h^{-}\right)$ and by Lemma 2 ,

$$
\begin{aligned}
\|2 f\| & \leq\left\|g^{+}\right\|+\left\|h^{+}\right\|+\left\|g^{-}\right\|+\left\|h^{-}\right\| \\
& =\|g\|+\|h\|=2=\|2 f\| .
\end{aligned}
$$

It follows from Lemma 3 that $g^{+}+h^{+}=2 f$ and $g^{-}=h^{-}=0$.

Now we show $g=h=f$, i.e., $f \in \operatorname{ext} B\left(X^{*}\right)$. This follows if we prove that $g(y)=h(y)=0$ whenever $f(y)=0$ (by [7, 11.5 , Theorem 1], this means $f=a g=b h$, but $f, g, h \in S\left(X^{*}\right)$ and $2 f=g+h$, so $\left.a=b=1\right)$. First we assume $y \geq 0$; then from $g(y) \geq 0, h(y) \geq 0$, and $g(y)+h(y)=2 f(y)=0$ we have $g(y)=h(y)=0$. For the general case, since $f(y)=0$ and by the condition given in the theorem, $f\left(y^{+}\right) f\left(y^{-}\right)=0$, we have $f\left(y^{+}\right)=f\left(y^{-}\right)=0$. Hence, $g(y)=h(y)=0$ follows from the first case.

Necessity. If there exist $x, y \in X$ satisfying $x \wedge y=0$ but $f(x)>0$ and $f(y)>0$, then we set $E=y^{\perp}$, and then by [1] $X=E+E^{\perp}$. Let $g=\left.f\right|_{E}$ and $h=\left.f\right|_{E^{\perp}}$. Then $\|g\|>0,\|h\|>0$ since $x \in E, y \in E^{\perp}$. Therefore, from

$$
f=\|g\| \frac{g}{\|g\|}+\|h\| \frac{h}{\|h\|}
$$

and $\|g\|+\|h\|=\|f\|=1$ according to Lemma 2, we see $f \in \operatorname{ext} B\left(X^{*}\right)$.

\section{WEAK CONVERGENCE AND WEAK COMPACTNESS IN ABSTRACT $M$ SPACES}

We begin with a lemma.

Lemma 10. Let $X$ be a $\sigma$ complete lattice. Then for any $x_{1}, \ldots, x_{m} \in X, X$ can be decomposed into $m$ many pairwise orthogonal subspaces. $X=E_{1}+\cdots+$ $E_{m}$ such that $\left.\left(x_{n}-\Lambda_{1 \leq m}\left(x_{i}\right)\right)\right|_{E_{n}}=0,1 \leq n \leq m$.

Proof. Since for any $x, y, z \in X,(x-z) \wedge(y-z)=x \wedge y-z$, replacing $z$ by $x \wedge y$, we obtain

$$
(x-x \wedge y) \perp(y-x \wedge y) .
$$

Set $\Lambda_{1 \leq n \leq m}\left(x_{n}\right)=x^{\prime}$ and $E_{1}=\left(x_{1}-x^{\prime}\right)^{\perp}$. Then by [1], $X=E_{1}+E_{1}^{\perp}$. Moreover, replacing $x, y$ by $x_{1}, \Lambda_{2 \leq n \leq m}\left(x_{n}\right)$ in $(*)$ respectively, we see

$$
\left.\left(x_{1}-x^{\prime}\right)\right|_{E_{1}}=0,\left.\quad\left(\bigwedge_{2 \leq n \leq m}\left(x_{n}\right)-x^{\prime}\right)\right|_{E_{1}^{\perp}}=0 .
$$

Let $E_{2}=\left\{x \in E_{1}^{\perp}:\left.x \perp\left(x_{2}-x^{\prime}\right)\right|_{E_{1}^{\perp}}\right)$. Then we also have $E_{1}^{\perp}=E_{2}+E_{2}^{\perp} \cap E_{1}^{\perp}$. Again by $(*)$ (replace $x, y$ by $\left.x_{2}\right|_{E_{1}^{\perp}}, \bigwedge_{3 \leq n \leq m}\left(x_{n}\right)$ respectively there), we have

$$
\left.\left(x_{2}-x^{\prime}\right)\right|_{E_{2}}=0,\left.\quad\left(\bigwedge_{3 \leq n \leq m}\left(x_{n}\right)-x^{\prime}\right)\right|_{E_{2}^{\perp}}=0 .
$$


And so on, we find pairwise orthogonal subspaces $E_{1}, \ldots, E_{m-1}, E_{m}=E_{m-1}^{\perp} \cap$ $E_{m-2}^{\perp}$ of $X$ such that $X=E_{1}+\cdots+E_{m}$ and $\left.\left(x_{n}-x^{\prime}\right)\right|_{E_{n}}=0, n \leq m$.

Theorem 11. Let $X \in A M$ be $\sigma$ complete. Then $x_{n} \rightarrow 0$ weakly in $X$ iff $\left\{x_{n}\right\}$ is bounded and $\lim _{m \rightarrow \infty}\left\|\Lambda_{i \leq m}\left(\left|x_{n_{i}}\right|\right)\right\|=0$ for all subsequences $\left\{x_{n_{i}}\right\}$ of $\left\{x_{n}\right\}$.

Proof. Sufficiency. If $\left\{x_{n}\right\}$ does not converge to zero weakly, then by the Rainwater Theorem there exist some $f \in \operatorname{ext} B\left(X^{*}\right), \varepsilon>0$, and a subsequence of $\left\{x_{n}\right\}$, again denoted by $\left\{x_{n}\right\}$, such that $f\left(x_{n}\right)>\varepsilon$ for all $n \geq 1$. Since by the proof of Theorem $9, f^{+}=0$ or $f^{-}=0$ and $f\left(x_{n}\right)=f^{+}\left(x_{n}^{+}\right)+f^{-}\left(x_{n}^{-}\right)-$ $f^{-}\left(x_{n}^{+}\right)-f^{+}\left(x_{n}^{-}\right)$, without loss of generality, we may assume $f \geq 0$ and $x_{n} \geq 0$ for all $n \geq 1$. Choose $m \geq 1$ such that $\left\|\Lambda_{n \leq m}\left(x_{n}\right)\right\|<\varepsilon$. Then by Lemma $10, X$ can be decomposed into the direct sum of pairwise orthogonal subspaces $E_{1}, \ldots, E_{m}$ such that $\left.x_{n}\right|_{E_{n}}=\left.x^{\prime}\right|_{E_{n}}$ for all $n \leq m$, where $x^{\prime}=\Lambda_{n \leq m}\left(x_{n}\right)$. By Theorem 9, there exists some $n \leq m$ such that $f=\left.f\right|_{E_{n}}$ which leads to a contradiction that

$$
\varepsilon<f\left(x_{n}\right)=f\left(\left.x_{n}\right|_{E_{n}}\right)=f\left(\left.x^{\prime}\right|_{E_{n}}\right) \leq\|f\| \cdot\left\|x^{\prime}\right\|<\varepsilon .
$$

Necessity. Suppose that $x_{n} \rightarrow 0$ weakly in $X$. If the condition is not necessary, then there exist a constant $\varepsilon>0$ and a subsequence of $\left\{x_{n}\right\}$, again denoted by $\left\{x_{n}\right\}$, satisfying $\left\|\Lambda_{n \leq m}\left(\left|x_{n}\right|\right)\right\|>2 \varepsilon$ for all $m \geq 1$. We first define $y_{1}^{1}=x_{1}^{+}$and $y_{2}^{1}=x_{1}^{-}$. Suppose that $\left\{y_{s}^{k}: s \leq 2^{k}, k \leq m\right\}$ have already been defined. Then we set $y_{2 s-1}^{m+1}=y_{s}^{m} \wedge x_{m+1}^{+}$and $y_{2 s}^{m+1}=y_{s}^{m} \wedge x_{m+1}^{-}$. By induction, we find $\left\{y_{i}^{m}\right\}$ satisfying $y_{i}^{m} \wedge y_{j}^{m}=0$ for all $m \geq 1$ and all $i, j \leq 2^{m}$ with $i \neq j$, and moreover, for any $k \leq m$, we have either $x_{k}^{+} \wedge y_{s}^{m}=0$ or $x_{k}^{-} \wedge y_{s}^{m}=0$ for each $s=1,2, \ldots, 2^{m}$. Hence, if we pick $j \leq 2^{m}$ such that $z_{m}=y_{j}^{m}$ satisfies $\left\|z_{m}\right\|=\max _{j \leq 2^{m}}\left\|y_{j}^{m}\right\|$, then

$$
\left\|z_{m}\right\|=\left\|\sum_{i \leq 2^{m}} y_{i}^{m}\right\|=\left\|\bigwedge_{n \leq 2^{m}}\left(x_{n}\right)\right\|>2 \varepsilon .
$$

Next, we select $f_{m} \in S\left(X^{*}\right)$ such that $f_{m}\left(z_{m}\right)=\left\|z_{m}\right\|$. Since $z_{m} \geq 0$ and $X^{*} \in A L$, we must have $f_{m} \geq 0$. In view of the Alaoglu Theorem [6], $\left\{f_{m}\right\}$ has a $w^{*}$-cluster $f \in B\left(X^{*}\right)$. It follows that for each fixed $n \geq 1$, we can find some $m \geq n$ such that $\left|f\left(x_{n}\right)-f_{m}\left(x_{n}\right)\right|<\varepsilon$. Let $F_{m}=z_{m}^{\perp}$ and $E_{m}=F_{m}^{\perp}$. Then $X=E_{m}+F_{m}$ by [1]. Note that $X^{*} \in A L$ implies $\left\|f_{m}\right\|=\left\|\left.f_{m}\right|_{E_{m}}\right\|+\left\|\left.f_{m}\right|_{F_{m}}\right\|$; from the fact

$$
1 \geq\left\|\left.f_{m}\right|_{E_{m}}\right\| \geq f_{m}\left(\frac{z_{m}}{\left\|z_{m}\right\|}\right)=1
$$

we see $\left\|\left.f_{m}\right|_{F_{m}}\right\|=0$. Since by the choice of $z_{m}, m \geq n$ implies that $x_{n}^{+} \wedge z_{m}=$ 0 or $x_{n}^{-} \wedge z_{m}=0$, we may assume $x_{n}^{+} \wedge z_{m}=0$. Thus, $\left.x_{n}^{-}\right|_{E_{m}} \geq\left. z_{m}\right|_{E_{m}}$, and so

$$
\begin{aligned}
\left|f\left(x_{n}\right)\right| & \geq\left|f_{m}\left(x_{n}\right)\right|-\left|f\left(x_{n}\right)-f_{m}\left(x_{n}\right)\right| \\
& >\left|f_{m}\left(x_{n}\right)\right|-\varepsilon=\left|f_{m}\right| E_{m}\left(x_{n}\right) \mid-\varepsilon \\
& \geq\left. f_{m}\right|_{E_{m}}\left(z_{m}\right)-\varepsilon=f_{m}\left(z_{m}\right)-\varepsilon \\
& =\left\|z_{m}\right\|-\varepsilon>\varepsilon,
\end{aligned}
$$

which contradicts the hypothesis that $x_{n} \rightarrow 0$ weakly. 
Theorem 12. Let $X$ be a dual $\sigma$ complete $A M$ space. Then a bounded subset $A$ of $X$ is weakly compact iff

$$
\sup _{\left(x_{n}\right) \subset A} \lim _{m \rightarrow \infty} \inf _{x \in K}\left\|\bigwedge_{n \leq m}\left(\left|x_{n}-x\right|\right)\right\|=0
$$

where $K=K\left(x_{n}\right)$ is the set of sequentially $w^{*}$-clusters of $\left\{x_{n}\right\}$ and, as usual, we denote $\inf \{r: r \in E\}=+\infty$ for $E=\varnothing$.

Proof. Necessity. Let $A$ be a weakly compact subset of $X$. Then for any sequence $\left\{x_{n}\right\}$ in $A$ we can pick a subsequence $\left\{x_{n_{i}}\right\}$ of $\left\{x_{n}\right\}$ weakly convergent to some point $x$ in $X$ and then obviously $x \in K=K\left(x_{n}\right)$. Therefore, it follows from Theorem 11

$$
\begin{aligned}
0 & =\lim _{m \rightarrow \infty}\left\|\bigwedge_{i \leq m}\left(\left|x_{n_{i}}-x\right|\right)\right\| \\
& \geq \lim _{m \rightarrow \infty}\left\|\bigwedge_{n \leq m}\left(\left|x_{n}-x\right|\right)\right\| \\
& \geq \lim _{m \rightarrow \infty} \inf _{y \in K}\left\|\bigwedge_{n \leq m}\left(\left|x_{n}-y\right|\right)\right\| \geq 0 .
\end{aligned}
$$

Sufficiency. For any sequence $\left\{x_{n}\right\}$ in $A$, by the given condition, $K=$ $K\left(x_{n}\right) \neq \varnothing$, hence, $\left\{x_{n}\right\}$ contains a subsequence, again denoted by $\left\{x_{n}\right\}, w^{*}$ convergent to some point $x \in K$. Hence, for any subsequence $\left\{x_{n_{i}}\right\}$ of this subsequence, $K^{\prime}=K\left(x_{n_{i}}\right)=\{x\}$ implies

$$
\lim _{m \rightarrow \infty}\left\|\bigwedge_{i \leq m}\left(\left|x_{n_{i}}-x\right|\right)\right\|=\lim _{m \rightarrow \infty} \inf _{y \in K^{\prime}}\left\|\bigwedge_{i \leq m}\left(\mid x_{n_{i}}-y\right)\right\|=0 .
$$

By Theorem 11, $x_{n} \rightarrow x$ weakly.

Remark 1. Replacing $X$ in Theorem 11 or Theorem 12 by $L_{\infty}$ or $l_{\infty}$, we obtain criteria of weak convergence and weak compactness for those spaces. But for $X=l_{\infty}$, since $w^{*}$-convergence of a bounded sequence in $X$ coincides with convergence in coordinates, which is also equivalent to weak convergence in $X=c_{0}$, we can prove, without any difficulties, the following corollary and from which one can easily deduce the relative results given in [8].

Corollary 13. A bounded subset $A$ of $l_{\infty}$ or $c_{0}$ is weakly compact iff

$$
\sup _{\left(x_{n}\right) \subset A} \lim _{m \rightarrow \infty}\left\|\liminf _{k \rightarrow \infty} \min _{n \leq m}\left(\left|x_{n}-x_{k}\right|\right)\right\|=0 .
$$

Remark 2. By [1], if an $A M$ space $X$ has a strong unit $e$, i.e., $x \in B(X)$ if and only if $|x| \leq e$, then $X$ is order isometric to a $C(K)$ space for an appropriate compact Hausdorff space $K$. However, in this paper, the $A M$ spaces are not assumed to have any units.

\section{REFERENCES}

1. J. Lindenstrauss and L. Tzafriri, Classical Banach spaces. II, Springer-Verlag, Berlin and New York, 1979.

2. W. A. J. Luxemburg and A. C. Zaanen, Riesz spaces. I, North-Holland, Amsterdam and London, 1971.

3. A. C. Zaanen, Riesz spaces. II, North-Holland, Amsterdam, New York, and Oxford, 1983.

4. L. V. Kantorovitch, B. Z. Vulih, and A. G. Pinsker, Functional analysis in partially order spaces, Gosudarstr, Izdat. Tehn. Teor. Lit., Moscow and Leningrad, 1950. (Russian) 
5. K. Yosida, Functional analysis, Springer-Verlag, Berlin and New York, 1978.

6. J. Diestel, Sequences and series in Banach spaces, Springer-Verlag, Berlin and New York, 1984.

7. A. Wilansky, Modern methods in topological vector spaces, McGraw-Hill, New York, 1987.

8. N. Dunford and J. T. Schwartz, Linear operators, Interscience, New York, 1958.

Department of Mathematics, Harbin Normal University, Harbin, China

Current address: Department of Mathematics, The University of Iowa, Iowa City, Iowa 52242

E-mail address: schen@math.uiowa.edu

Department of Mathematics, Harbin Institute of Technology, Harbin, China

Current address: Department of Mathematics, University of Memphis, Memphis, Tennessee 38152

E-mail address: sunh@hermes.msci.memst .edu 